\title{
Redes Neurais: Uma Aplicação na Previsão de Vendas
}

\author{
Angela P. Ansuj \\ Maria Emília Camargo \\ Deoclécio Gomes Petry
}

Programa de Pós-Graduação em Métodos Quantitativos

Departamento de Estatística - Centro de Ciências Naturais e Exatas - UFSM

Santa Maria, RS.

Palavras Chave: Rede Neural, ARIMA, Retropopagação

\section{RESUMO}

Aplicou-se os modelos ARIMA e de retropropagação na análise do comportamento da série de vendas de uma empresa de porte médio de Santa Maria, no periodo de janeiro de 1979 a dezembro de 1989 . Inicialmente, avaliou-se os dados a partir de uma análise exploratória e das funções de autocorrelação e de autocorrelação parcial, com o objetivo de verificar a existéncia de componentes sazonais, de não estacionaridade e aleatoriedade dos dados. $O$ número de unidades da camada intermediária foi determinado por tentalivas. Utilizou-se a análise da sćrie residual para detcrminar o modelo mais adeçuado aos dados, bem como para escolher a melhor rede. $A$ previsão pontual obtida alravés da rede Neural toi supcrior ao modelo ARIMA de Box-Jenkins. 


\section{Introdução}

Neste trabalho, utilizou-se modelos ARIMA de Box-Jenkins e o de Retropropagação, que consiste de uma Rede Neural multicamada com as unidades conectadas apenas com as unidades da camada subsequente e com a informação passada em única direção, (BoxJenkins, 1976; CAMARGO, 1992; WASSERMAM, 1989) para o comportamento de uma série de vendas mensal de uma empresa de porte médio de Santa Maria, Rio Grande do Sul, no periodo de janeiro de 1979 à dezembro de 1989.

\section{Metodologias}

\section{Modelo ARIMA com intervenção}

A análise de intervenção é um modelo de Função de Transferência Estocástica, onde é possivel interpretar a maneira de incorporar seus efeitos ao modelo da série temporal.

Os maiores efeitos da intervenção são notados na mudança do nível na direção ou inclinação da série em estudo, e também para alterar as variáveis dos erros e introduzir no modelo componentes que não haviam antes; regressivos em um processo de médias móveis.

Seja uma série temporal para a qual verifīcou-se e estimou-se um modelo ARIMA com o qual vêm se fazendo previsões há algum tempo; num dado instante ocorre um evento independente do fenômeno que originou a série temporal, mas cujos efeitos podem se manifestar sobre ela.
Esse evento externo, cujos efeitos influenciam a série em estudo, deve ser incorporado ao modelo, como uma informação adicional à série. Esta incorporação de informação chama-se de intervenção. O modelo de intervenção pode ser representado por:

$$
\begin{aligned}
& f(k, x, t)=\sum_{\mathrm{j}=1}^{\mathrm{k}} v_{j}(B) x_{\mathrm{t} j}+\eta_{\mathrm{t}} \\
& f(k, x, t)=\sum_{\mathrm{j}=1}^{\mathrm{k}} \frac{\mathrm{w}_{j}(B)}{\sigma_{j}(B)} x_{\mathrm{tj}}+\eta_{\mathrm{t}}
\end{aligned}
$$

$\mathrm{x}_{\mathrm{t}, \mathrm{j}}, \mathrm{j}=1,2, \ldots ., \mathrm{k}$ são variáveis exógenas (intervenções). Eventualmente pode-se usar $x_{t-b}$, onde $b$ é o espaço de tempo decorrido entre as séries de entrada e saida (defasagem entre as séries); $k$ é o conjunto de parâmetros desconhecidos que aparecem em $v_{j}(B)$ ou em $\mathrm{w}_{\mathrm{j}}(\mathrm{B}) \mathrm{e}_{\mathrm{j}}$ (B).

Onde: $v_{j}(B)=w_{j}(B) /{ }_{j}(B), j=1,2, \ldots, k$ é a função de transferência da $j$-ésima variável exógena, sendo $v_{j}(B), w_{j}(B) e_{j}(B)$ polinômios em $B$ e é o ruído que pode ser representado por um modelo ARIMA, inclusive com componentes sazonais.

Cada série $x_{i j}$ é um indicador que assume os valores 0 ou 1 , representando, respectivamente, a ausência ou a presença da j-ésima intervenção, isto é, a não ocorrência ou a ocorrência do j-ésimo evento.

As séries indicadoras de intervenções podem ser representadas por três tipos de variáveis binárias.

1) Função Impulso - Intervenção do tipo 1;

2) Função degrau ("Step function") - Intervenção tipo 2;

3) Função impulso sazonal - Intervenção tipo 3. 


\section{Modelo de Rede Neural}

O modelo de Retropropagação é o paradigma comumente usado em áreas como reconhecimento de sinais, e principalmente em Previsão de Séries Temporais (BEALE, 1990 e MELO, 1991). O modelo retropropagação utiliza uma topologia de 3 ou mais camadas. As conexões entre as unidades são do tipo intercamadas e são direcionadas da camada de entrada para a camada de saída.

A rede neural se ajusta como um modelo de previsão de séries temporais da seguinte forma:

1 - as unidades de entrada são compostas por informações relevantes para a previsão;

2 - os pesos são os parâmetros do modelo e serão estimados através do aprendizado da rede que leva em conta pares de entradas com as respectivas saidas alvos (valores reais da série temporal);

3 - as unidades da camada escondida servem de ligação entre as camadas e entrada e saida; seu papel é fundamental para a escolha do melhor conjunto de pesos, pois a não linearidade do modelo se localiza na função de ativação das unidades escondidas;

4 - a camada de saida é composta de uma única unidade e portadora da informação desejada: a previsão;

5 - o treinamento dos pesos é a estimação dos parâmetros do modelo; como o aprendizado é supervisionado, podemos, a partir do treinamento, gerar na camada de saida previsões de $1,2, \ldots$, etc., passos à frente.

6-Os hiperparâmetros são valores forneci- dos pelo usuário, que em geral são constantes. São eles: taxa de aprendizado, termo de momento e tamanho do intervalo de variação dos pesos e tendências.

No modelo de retropropagação os parâmetros (pesos das conexões e tendências) são modificados durante o treinamento pelo método gradiente. No modelo de retropropagação original, a função de custo é tomada como a variância residual total, ou soma total dos quadrados para uma série de pontos $\mathrm{S}$.

$$
\text { tss }(S)=\left(\text { real }_{k}-\text { previsão }_{k}\right)^{2}=\left(x_{1}-x_{1}\right)^{2}
$$

onde $\mathrm{x}_{1}$ é o valor real da série temporal $\mathrm{t}, \mathrm{e}$ $\mathrm{x}_{1}$ é o valor de saída da rede para o tempo $t$. Essa medida indica o quanto bem a superficie sobre o espaço de entrada se ajusta aos pontos $\left(x_{t}, t\right.$ e $\left.S\right)$. O valor de MSE (Mean Square Error)é obtido dividindo-se o valor de tss pelo tamanho do conjunto,

$$
\mathrm{MSE}=\frac{\mathrm{tsS}}{\mathrm{S}}
$$

o valor de RMSE (Root Mean Square Error) é obtido por:

$$
\text { RMSE }=\sqrt{\mathrm{MSE}}
$$

$e$, finalmente, o valor de nRMSE é obtido dividindo-se o valor de RMSE pelo desvio padrão da série para obtermos um valor que permita a comparação entre séries distintas;

$$
\mathrm{nRMSE}=\frac{\mathrm{RMSE}}{\sigma_{\mathrm{s}}}
$$

arv (average relative variance) $=\mathrm{nRMSE}^{2}$ 


\section{Modelos estimados}

\section{Análise Preliminar}

Inicialmente avaliou-se os dados a partir de uma análise exploratória e das funções de autocorrelação e de autocorrelação parcial, verificando-se a existência de componentes sazonais, de não estacionariedade e aleatoriedade dos dados.

\section{Modelo ARIMA com intervenção}

O modelo ajustado com intervenção ARIMA $(1,1,6)(0,1,0,)_{12}$, cujos parâmetros estimados e as estatísticas estão apresentados na TABELA 1.

\begin{tabular}{c|c|c}
\hline \hline Parâmetro & Valor Estimado & Estatística “t” \\
\hline$\phi 1$ & -0.37774 & -4.05 \\
$\boldsymbol{\theta} 6$ & -056638 & -6.45 \\
$\xi 1$ & -0.14228 & -4.93 \\
$\xi 2$ & -0.22293 & -5.06 \\
$\xi 3$ & -0.17447 & -4.21 \\
$\xi 4$ & -0.11857 & 3.37 \\
$\xi 5$ & -0.99164 & 2.99 \\
\hline
\end{tabular}

TABELA 1 - Parâmetros estimados e estatística " $t$ " para o modelo univariado com intervenção para a série de vendas de uma empresa de porte médio de Santa Maria

As estatísticas de ajuste para o modelo são:

$$
\mathrm{R}^{2}=0.98015
$$

$\mathrm{AIC}=-5.9484$

$\mathrm{BIC}=-5.7841$

As estatísticas do ruido são:
Média $=-0.00708 \quad 0.00$

$$
\text { Desvio Padrão }=0.04762
$$

$$
\text { Variância }=0.00227
$$

As intervenções identificadas para a série de Vendas durante o período analisado, a um nível de significância de 5\% estão apresentados na TABELA 2.

\begin{tabular}{c|c|c}
\hline \hline Tipo de Intervenção & Instante & Período \\
\hline$X_{11} \rightarrow 1$ & 99 & MAR/87 \\
$X_{2 t} \rightarrow 1$ & 131 & NOV/89 \\
$X_{31} \rightarrow 2$ & 123 & MAR/89 \\
$X_{4 t} \rightarrow 3$ & $115,127, \ldots$ & JUN/88 \\
$X_{5 t} \rightarrow 2$ & 108 & DEZ/87 \\
\hline
\end{tabular}

TABELA 2 - Tipos de Intervenção detectadas

As intervenções ocorridas são do tipo impulso, degrau e impulso sazonal.

Observa-se que as estimativas dos coeficientes das variáveis de intervenção " $i$ " tem os sinais esperados. Isto é, ${ }_{1} e_{2}$ têm sinais negativos, enquanto ${ }_{3}{ }_{4} \mathrm{e}_{5}$ têm sinais positivos.

A inclusão destas variáveis no modelo pode ser justificada da seguinte forma:

1) a primeira intervenção representa os reflexos, devido ao plano cruzado que impôs um congelamento de preços, o qual vigorou de março a novembro de 1986 ;

2) a intervenção de novembro de 1989 é o reflexo do choque heterodoxo do plano verão;

3) a intervenção ocorrida em março de 1989 é devido ao acréscimo dos preços em 
conseqüência da memória inflacionária;

4) o acréscimo das vendas em junho de 1988 , é caracterizado pelo efeito sazonal, pois em todos os anos, a partir de 1988, tem ocorrido acréscimo nas vendas, sendo mais acentuado no ano de 1988; e como pode ser observado, a harmonia referente ao mês de junho é altamente significativa;

5) fïnalmente, esta variável representa a intervenção devida ao Plano Bresser, uma nova tentativa de congelamento de preços, desta vez por um período mais curto, de julho a outubro de 1987.

\section{Rede Neural}

\section{Arquitetura}

Foram testadas várias arquiteturas e avalisadas as previsões, sendo que a arquitetura escolhida foi:

i) 14 unidades na camada de entrada, da seguinte forma:

- 2 lags passados: $\mathrm{x}_{\mathrm{t}}$ e $\mathrm{x}_{\mathrm{t}-1}$;

-12 unidades sazonais

$$
\begin{aligned}
& \begin{array}{llllll}
1 & 0 & 0 & 0 \ldots & 0
\end{array} \\
& \begin{array}{lllll}
0 & 1 & 0 & 0 \ldots & 0
\end{array} \\
& \begin{array}{llllll}
0 & 0 & 1 & 0 \ldots & 0
\end{array} \\
& \begin{array}{llllll}
0 & 0 & 0 & 1 \ldots & 0
\end{array} \\
& \begin{array}{lllll}
0 & 0 & 0 & 0 \ldots .
\end{array}
\end{aligned}
$$

ii) duas unidades na camada escondida; iii) uma unidade na camada de saída $x_{t+1}$

\section{Treinamento}

O treinamento foi repetido 1950 vezes, com as atualizações dos pesos realizadas a cada 20 padrões apresentados sem fermentação.

A constante de aprendizado foi mantida igual a 0,14 e, nas últimas 400 vezes, usou-se o termo de esquecimento igual a 0,5. Este valor foi considerado a fim de se dar mais peso às observações mais recentes.

O termo momento usado foi de 0,8 e o tamanho do intervalo de variação dos pesos foi igual a 4 .

\section{Comparação do Modelo ARIMA e da Rede Neural.}

O modelo ARIMA com intervenção apresentou uma variação residual de 0.00227 , enquanto o modelo da rede neural apresentou uma variância residual de 0.00104 .

A rede neural escolhida apresentou, para os últimos 12 meses, as melhores previsões que o modelo ARIMA com intervenções. $O$ erro absoluto médio de previsão foi de 7.6486 para o modelo obtido pela rede, enquanto o modelo ARIMA com intervenções foi de 9.8642 .

\section{Conclusão}

A série de vendas apresentou uma sazonalidade marcante, para a qual foi necessário 12 unidades binárias ( 0 ou 1$)$ para determinar $o$ peso relativo e o mês. 
O modelo obtido pela rede neural foi superior ao modelo ARIMA, tanto no ajuste como na previsão.

\section{Referências Bibliográficas}

BOX, G. E. \& JENKINS, G. M. Time séries analysis, forecasting and control. San Francisco, Holden Day.

CAMARGO, M. E. Modelagem Clássica e Bayesiana: uma evidência empírica do processo inflacionário brasileiro. Tese de Doutorado. Programa de PósGraduação, UFSC, 1992.
MELO, M. P. (1991). Redes Neurais Artificiais: uma aplicação à previsão de preços de derivados de Petróleo. Dissertação de Mestrado. Depto de Informática. PUC-RJ.

WASSERMAN, P.D. (1989). Neural Compreting. Theory and practice. Van Nostrand Reinhold. 